\title{
Catheter placement for hemodialysis in a covid- 19 patient in the prone by anatomy, a case report
}

\begin{abstract}
Summary
The use of renal replacement therapies in patients with SARS-COV2 infection accounts for up to $20 \%$ of patients admitted to the intensive care unit. In patients who develop a severe ARDS, the American and European guidelines recommend the prone position in order to improve pulmonary ventilation. However, the placement of a hemodialysis catheter is a great challenge and the use of technology, such as ultrasound, may help to reduce complications. However, when it is not available, the importance of an anatomical knowledge is vital for its successful colocation.
\end{abstract}

Keywords: catheter, prone position, COVID-19, anatomic reference
Volume 14 Issue 5 - 202 I

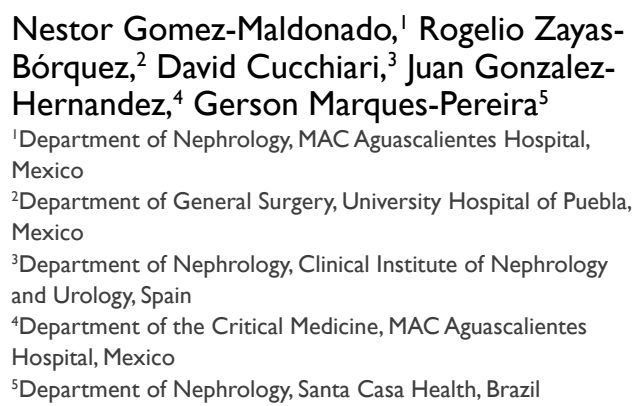

Correspondence: Rogelio Zayas Bórquez. Camino Real Cipreses 1404 Int 6 3, San Andres Cholula, Puebla, CP, 728I0, Mexico, Email Rogeliozb94@Gmail.com

Received: July 21, 202I | Published: September 06, 202

\section{Introduction}

The recent pandemic by the new virus SARS-COV 2, worldwide, has brought unimaginable amounts of challenges for clinicians in different areas. Since its appearance at the end of 2019, until the last report in August 2020, there have been 24,421,364 cases worldwide, reporting up to 831,764 deaths, according to reports from the CDC.

Patients with moderate to severe conditions often require ventilator mechanical support and vasopressors in an intensive care unit setting, and eventually may develop acute kidney injury with necessity of dialysis. ${ }^{1}$ Renal replacement therapy is one of the tools most used in patients with COVID-19, since $20-40 \%$ of patients admitted to an intensive care unit develop Acute kidney injury, and of these, up to $20 \%$ will require some method of renal replacement therapy, from conventional hemodialysis, to continuous slow therapies, mainly in those with hemodynamic compromiso..$^{2-4}$

As is well known, a fundamental part of adequate dialysis therapy, it is imperative to place a catheter that allows adequate flows treatment. Currently, the KDIGO guidelines recommend the following order for the placement of catheters: right internal jugular vein, femoral veins, left internal jugular vein, subclavian vein of the dominant arm, leaving the subclavian vein of the non-dominant arm as the last option. ${ }^{5}$ As part of the recommendations, the same guidelines recommend that for the placement of a catheter, this should be done through the guidance of an ultrasound. However, in many circumstances this may not be possible, so it is required to have a broad knowledge of the anatomical landmarks in order to properly locate the vessel to be cannulated.

On the other hand, as an integral part of the management in patients with SARS-COV2 pneumonia, who develop severe ARDS, both the American and European guidelines recommend the prone position. This has been associated with an improvement of ventilation-perfusion, increased lung volume at the end of expiration, and decreased volume-induced damage by a more even distribution of tidal volume. With some differences regarding the permanence of time in the prone position, the guides recommend an approximate time that goes from 12 to 16 hours of permanence. ${ }^{6-8}$

\section{Case presentation}

A 62-year-old male as admitted on July 23rd 2020 with nonproductive cough, asthenia, adynamia and dyspnea due to COVID-19 bilateral pneumonia that eventually needed ventilator mechanical support with high ventilator parameters. Therefore, as an added therapeutic measure, the patient was pronated, presenting ventilator improvement. After 6days of stay in the intensive care unit, renal compromise deterioration occurred in form of oliguric AKIN 3 acute kidney injury, without response to diuretics, making dialysis start necessary as a life-saving procedure. Because the patient's requirements to maintain a prone position, the decision was to place a temporary double-lumen catheter in the same position, in the absence of ultrasound support, and using anatomical references to guide colocation.

\section{Description of the technique}

After a comprehensive explanation of the risks and benefits, in addition to the signing of informed consent by the responsible family members, fields and sterile material are set in place.

In the prone position, asepsis and antisepsis of the left jugular region are performed, hyperextension of the neck and discrete traction of the ipsilateral arm are performed, to widely delimit the sternocleidomastoid muscle. The posterior approach chosen consisted of superficial infiltration of simple $1 \%$ lidocaine on the lateral border of the clavicular insertion of the sternocleidomastoid muscle, approximately $3 \mathrm{~cm}$ from the left clavicle, with an angulation of $30^{\circ}$, towards the ipsilateral teat Once infiltration has been performed, in order to minimize the risks, the jugular vein is located using a 22 $\mathrm{G}$ needle, obtaining venous flow on the first attempt. After that, the 
syringe is withdrawn, carefully fixing the needle to later use it as a reference and direct the trocar in parallel. After obtaining venous flow through the trocar, the $22 \mathrm{G}$ needle is withdrawn, and subsequently the trocar syringe is used to pass the metal guide, which flows without resistance, with the usual Seldinger technique. After withdrawing the trocar and dilators are passed, a temporary catheter with a double lumen of $11.5 \mathrm{Fr}$ and $19.5 \mathrm{~cm}$ length is collocated, confirming adequate flow of both lumens. After that, the two branches of the catheter are heparinized, fixed with 2-0 nylon, and covered with sterile material, and the procedure is terminated without eventualities. The patient later underwent an extended hemodialysis session, which ended without complications.

\section{Discussion}

In patients with severe ARDS who need prone position and develop acute kidney injury, the placement of an adequate catheter is a vital procedure that allows the start of renal replacement therapy, either through continuous slow therapy or conventional hemodialysis. Although the current guidelines, both KDIGO and KDOQI, widely recommend the use of ultrasound for the colocation of these catheters, it is important to have adequate anatomical knowledge in case. ${ }^{5}$ This tool is not available. This skill will allow to find of a suitable blood vessel to place the catheter and to reduce the own risks that this technique entails.

\section{Conclusion}

It is therefore that the technique described in this article provides one more therapeutic alternative that can be used in atypical situations such as the one we live in. A review of this case was carried out to underline the importance of anatomical knowledge that can be used in the intensive care setting when technology is not readily available.

\section{Acknowledgments}

None.

\section{Conflicts of interest}

Author declares there are no conflicts of interest.

\section{Funding}

There was no funding for this report.

\section{References}

1. Karkar A, Ronco C. Prescription of CRRT: a pathway to optimize therapy. Ann Intensive Care. 2020;10(1):32.

2. Durvasula R, Wellington T, McNamara E, et al. COVID-19 and Kidney Failure in the Acute Care Setting: Our Experience From Seattle. Am J Kidney Dis. 2020;76 (1):4-6.

3. Ronco C, Reis T, Husain-Syed. Management of acute kidney injury in patients with COVID-19. Lancet Respir Med. 2020;8(7):788-742.

4. Ronco C, Bangshaw S, Bellomo R, et al. Extacorporeal Blood Purifiation and Organ Support in the Critically III Patient durin COVID-19 Pandemic: Expert Review an Recommendation. Blood Purif. 2021;50(1):17-27.

5. Khwaja A. KDIGO clinical practice guidelines for acute kidney injury. Nephron Clin Pract. 2012;120(4):c179-c184.

6. Gonzalez F, Salme L, Olvera C. Posición prono en pacientes con síndrome de insuficiencia respiratoria progresiva aguda por COVID-19. Med Crit. 2020;34(1):73-77.

7. World Health Organization(WHO). Clinical Management Of Severe Acute Respiratory Infection (SARI) when COVID-19 disease is suspected. Geneva: World Health Organization(WHO); 2020.

8. Fan E, Del Sorbo L, Goligher EC, et al. An official American Thoracic Society/European Society of Intensive Care Medicine/Society of Critical Care. Medicine Clinical Practice Guideline: Mechanical Ventilation In Adult Patients With Acute Respiratory Distress Syndrome. Am J Respir Crit Care Med. 2017;195(9):1253-1263. 